Mechanisms

\section{of Virus Persistence}

Richard T. Johnson, MD,* Robert A. Lazzarini, PhD, $\uparrow$ and Byron H. Waksman, MD

Circumstantial evidence has related multiple sclerosis to a possible viral infection. However, any hypothetical mechanism must be consistent with known epidemiological, clinical, and pathological data. Therefore, if a virus is involved, the infection must have a long incubation period, cause remitting and relapsing disease, and lead to demyelination by one of various mechanisms. A conference sponsored by the $\mathrm{Na}$ tional Multiple Sclerosis Society on June 7 and 8, 1980, in Aspen, Colorado, focused on mechanisms of viral persistence with particular emphasis on the central nervous system. The conference, chaired by R. T. Johnson and R. A. Lazzarini, brought together 50 investigators from eight countries, including neurobiologists, molecular biologists, animal virologists, neurologists, and neuropathologists. Discussions dealt with the relevance of viral infection to multiple sclerosis, the biology of static neural cell populations that pose restraints on some mechanisms of persistence and enhance others, and the varied mechanisms of viral persistence that may operate in the central nervous system.

Some viruses are known to persist by preservation of viral DNA in host cells. Recent studies indicate that the DNA of herpesviruses and the proviral forms of some retroviruses (viruses which contain a reverse transcriptase) are the long-term repositories of genetic information in neural cells persistently infected with these viruses. Herpes simplex virus remains latent in sensory ganglia of both humans and animals, and animal studies indicate that the latency is in ganglion cells, not satellite cells. Viral DNA can persist by integration with host cell genes, but integration has long been assumed to accompany host DNA synthesis. In nondividing neurons, integration might occur during the process of DNA repair synthesis. However, recent evidence suggests that preservation of viral DNA does not always require integration. Indeed, in Epstein-Barr virus infections of B

From the *Department of Neurology, Traylor 709, The Johns Hopkins University School of Medicine, Baltimore, MD 21205 , the Haboratory of Molecular Biology, National Institute of Neurological Communicative Disorders and Stroke, Bethesda, MD 20205 , and the \pm National Multiple Sclerosis Society, New York, NY.

Received Oct 20, 1980. Accepted for publication Oct 24, 1980. Address reprint requests to Dr Johnson. lymphocytes, much of the viral DNA is episomal. Studies of mutants of herpes simplex virus were presented which support the idea that host cell DNA synthesis is not essential for the establishment of latency; these studies also demonstrate that one or more early and one or more late viral gene functions are essential to establish latency. Host factors are also important, since administration of antibody of neurotransmitters may alter the latency of herpes simplex virus.

Retroviruses persist by the preservation of proviral DNA, which probably is integrated with host cell DNA, and integration is an apparent prerequisite to viral replication. In sheep chronically infected with visna virus, proviral DNA in the virus has been demonstrated in blood macrophages and cells of undefined origin in the brain. This virus, which causes inflammatory demyelinating disease, and related viruses responsible for chronic pneumonitis and arthritis, can relapse, possibly due to antigenic mutations which have been shown to develop over time in the presence of antibody. Viruses selected by this immunological pressure are mutated in the region of the virus genome, which presumably codes for the surface glycoprotein involved in virus neutralization. Mutants have been shown to be fully virulent, but they cannot be neutralized by antibody formed in the host in response to the initial virus.

Viruses such as the paramyxoviruses and coronaviruses are known to persist within the central nervous system under certain circumstances, and virtually all available evidence indicates that they do so by mechanisms which do not involve proviral DNA. Some of these budding viruses fail to assemble or release infectious virus, and this may be dependent on virus properties, immune responses, and host cell restrictions. The possibility is generally acknowledged that measles virus persistence in patients with subacute sclerosing panencephalitis (SSPE) may be related to an alteration in the metabolism of viral $M$ protein. The $M$ protein is essential to alignment of the nucleocapsids formed in cytoplasm with the viral glycoproteins inserted in the cytoplasmic membrane. The accumulation of unaligned nucleocapsids and the failure to bud and release infectious enveloped virus can be explained by this defect. Whether or not an immune response to $M$ protein is abrogated in patients with SSPE remains a subject of controversy, and the question of whether the low levels of anti-M protein antibodies in SSPE patients signifies restricted or limited synthesis of viral protein in brain cells is hotly debated. Comparisons of peptide maps of the $M$ proteins in strains of wild type measles and SSPE show variability, but no consistent abnormality has yet been uncovered to differentiate SSPE and wild measles viruses. 
Other abnormalities of replication can lead to persistence or slowing of the infectious process; examples include mutants of Newcastle disease virus in which abnormalities of the fusion protein can cause slow virus spread or in which less messenger RNA is produced and less cytopathological change occurs despite normal yields of infectious virus. Varying expression of the same virus in different cells lines was also demonstrated. In lymphoid lines infected with measles virus, some cell lines fail to cleave the virus fusion protein precursor and thus release noninfectious virus; conversely, the effect of virus persistent infection may vary in different cell types. In neuroblastoma cell lines chronically infected with coronaviruses, some cells show no change in cell growth, yet neurites could not be induced; and in neuroblastoma cell lines chronically infected with lymphocytic choriomeningitis virus, growth, protein, and DNA metabolism were normal but generation of transmitters was selectively inhibited.

Infection can also be modulated by interference caused by defective interfering particles, by interferon, or by immune responses. Defective interfering particles lack full genetic information and therefore replicate only with the help of standard infectious particles; however, their replication in turn suppresses the replication of standard particles. Defective interfering particles have the capacity to modulate or cycle production of virus in cell cultures, although this has not been demonstrated in vivo. The patient's immune response may also modulate rather than clear infection. Virus may escape from immune surveillance because of antibody-induced antigen modulation, production of blocking factors, or generation of suppressor $T$ cells. This may be of particular importance in the central nervous system, where spinal fluid levels of antibody and complement are both low and therefore may modulate infection in cells in the nervous system, where high levels may lyse cells and clear virus in the extraneural tissues.

Interferon may save cells from destruction by cytolytic viruses, and viral mutants that induce defective interfering particles or other virus mutants may induce interferon that spares the lethal effects and thereby enhances virus persistence.

It is evident that there are numerous mechanisms which channel infection into a chronic form, and these phenomena can act in unison, in sequence, or individually. Virus isolated from persistent infecrions usually is genetically altered, and a number of experiments suggest that the rate of spontaneous mutations among RNA viruses, which is normally high, may be increased in persistent infections. Mutations are most likely to affect the RNA-synthesizing components of the virus and thus lead to a cascade of mutations, similar to what was predicted by Orgel [1]. From the greatly increased diversity of progeny viruses thus generated, viruses are likely to be selected which are so enfeebled that they do nor kill the host cell but which, nevertheless, replicate fast enough so that large numbers of progeny cells remain infected. Viruses of this type are the hallmark of many persistent infections. Thus, error catastrophe may be involved in maintaining a persistent infection but is unlikely to have played a role in establishing it.

This conference was supported in part by the National Institute of Neurological and Communicarive Disorders and Stroke, the National Institute of Allergy and Infectious Diseases, the National Cancer Institute, and the John E. Fogarty International Center for Advanced Study in the Health Sciences.

\section{Reference}

1. Orgel LE: The maintenance of the accuracy of protein synthesis and its relevance to ageing. Proc Natl Acad Sci USA 49:517521,1963 